\title{
Los estudios de la comunicación más allá de la disciplina
}

\section{Communication Studies Beyond the Discipline}

\author{
Gabriel Barrón Pérez \\ (D) https://orcid.org/0000-0002-9266-305X \\ Departamento de Letras \\ Universidad de Guadalajara, México \\ gabrielbarronp@gmail.com
}

Resumen: En el marco de la frecuente preocupación que los estudiosos de la comunicación manifiestan respecto del lugar disciplinar que ocupan y de la relevancia que cobran los estudios socioculturales anclados en diferentes expresiones y tecnologías comunicacionales, el objetivo de este artículo es cuestionar teóricamente la disciplinarización de los estudios de la comunicación. El autor sondea el contradictorio empleo de los conceptos de "campo" y "mapa" como nociones que involucran la apertura disciplinar, examina las disciplinas como un ejercicio acotado del pensamiento y propone una imagen relacional, abierta y ontológica de la comunicación.

Palabras clave: estudios de la comunicación; disciplina; mapa; ilustración; espacio relacional.

Abstract: Within the framework of the frequent concern expressed by communication scholars regarding the disciplinary place they occupy and the relevance of sociocultural studies anchored in various communication expressions and communication technologies, the objective of this article is to theoretical-

cómo citar: Barrón Pérez, G. (2020). Los estudios de la comunicación más allá de la disciplina. Secuencia (108), e1739. DoI: https://doi.org/10.18234/secuencia.v0i108.1739

cc $(1)$ Esta obra está protegida bajo una Licencia Creative Commons Atribución-NoComercial 4.0 Internacional. 
ly question the discipline of communication studies. The author probes the contradictory use of the concepts of "field" and "map" as notions that involve disciplinary openness, examines disciplines as a limited exercise of thought and proposes a relational, open, and ontological image of communication.

Key words: communication studies; discipline; map; illustration; relational space.

Recibido: 12 de abril de 2019 Aceptado: 8 de enero de 2020

Publicado: 21 de agosto de 2020

La estructuración e institucionalización del campo de estudios de la cocuyas reflexiones se asumen o han sido asumidas como una indagación al respecto. Los fundamentos teóricos acerca de la noción de "campo" que formula Bourdieu (1976, 2012); el replanteamiento epistemológico de las ciencias sociales postulado por Wallerstein (2004); la crítica al predominio de una visión sistémica, funcional, mediática y acrítica de la comunicación de Pasquali (2008); los análisis sobre la carencia de canon disciplinario de la comunicación que elaboran Craig (1999), Vidales (2011), Fuentes (1998; 2003; 2010; 2011a; 2011b), Martín-Barbero (2010), Krippendorff (1994) o Peza (2013), y las descripciones de los objetos, epistemologías o tensiones teóricas al interior del campo que trazan Jensen (2012a, 2012b, 2012c), Moragas (2011), Zallo (2011) y Piñuel-Raigada (2011) son precisamente reflexiones que expresan la inquietud de la forma en que se construye, se valida y se insituye el conocimiento producido por los estudios de la comunicación dentro de los marcos generales de la ciencia y, más específicamente, de las ciencias sociales.

Esta serie de preocupaciones son indicadores de la necesidad de reconocerse en un lugar del orbe científico, de la dificultad para autorrepresentarse discursivamente y de una fuerte autocrítica que reluce en el trasfondo: los estudios de la comunicación carecen de una personalidad intelectual distintiva, clara y transmisible.

La radical importancia contemporánea de estudiar comunicativamente procesos de subjetivación y de reproducción de representaciones sociales y simbólicas ancladas en tecnologías digitales, la menoscabada jerarquía intelectual con que los estudios y los estudiosos de la comunicación enfren- 
tan el reto, y la oportunidad de reorientar su pensamiento y sus estrategias conceptuales en claves comunicativas, sociológicas, filosóficas o éticas, son tres condiciones que ofrecen la coyuntura para cuestionar a los estudios de la comunicación más allá de la restricción epistemológica y, finalmente institucional, de "campo" o "disciplina".

Con base en un conjunto de insumos teóricos de corte comunicacional, sociológico y filosófico, las siguientes páginas procuran dos objetivos. Por un lado, exponer un conjunto de ideas que animen la discusión en torno de la acotada imagen de los estudios de la comunicación respecto de los importantes retos que enfrenta; por otro, cuestionar teóricamente la tensión disciplinante que incorpora la noción de "campo" a partir de esbozar a la comunicación como un espacio relacional de saberes.

De este modo, en el primer apartado se presenta el contradictorio empleo de nociones como "mapa", "cartografía" o "campo" a manera de recursos conceptuales para examinar la disciplinariedad de las ciencias sociales y la comunicación; en el segundo, se describen algunas implicaciones oclusivas de lo disciplinar como resultado de la trama cartesiana ilustrada; en el último apartado se explora la idea de meditar a la comunicación como un espacio relacional del conocimiento.

\section{UNA ESPACIALIDAD ABIERTA PERO CERRADA}

El llamado a cuestionar los paradigmas de las ciencias sociales (Ortiz, 1999; Wallerstein, 2004), a replantear sus estatutos disciplinares (Vidales, 2011; Zallo, 2011), o a examinar a la inter, multi, trans o posdisciplinariedad como modelos de trabajo científico, en particular dentro de los estudios de la comunicación (Fuentes, 1998, 2002; Martín-Barbero, 2002; Reguillo, 2004), son variedades estructurales que análogamente apuntan a la necesidad histórica de criticar, abrir y desmantelar los objetos de estudio, marcos teóricos y supuestos epistémicos que la modernidad ilustrada construyó para alimentar la cientificidad de la reflexión sobre los fenómenos sociales durante el siglo XIX y buena parte del xx. Si bien el conjunto de autores y dimensiones estructurales distinguirían variantes de contenido en lo que respecta a la necesidad de una revuelta contra la disciplinarización del pensamiento, proyectan una notable correspondencia semántica en el ideal de abrir aquello que se manifiesta como un núcleo cerrado de saberes. Es por este ascendente que se dirige 
a la crítica o disolución de lo cerrado que resulta contradictorio que se emplee como una apuesta contra la cerrazón otro grupo de conceptos o ideales que refieren justo emplazamientos ("campo", "marco", "lugar") o metáforas ("cartografía", "mapa", "topografía") que aluden no sólo al espacio acotado y visual, sino al lugar que se ocupa dentro de las coordenadas que visibiliza. ${ }^{1}$

Afincada en la lógica argumentativa de Wittgenstein, Pitkin (1984) critica la ligereza con que en las ciencias sociales se sirven de ciertas palabras (su trabajo analiza los términos "poder" y "legitimidad") sin el necesario arbitrio de la reflexividad teórica o semántica. Tal parece ser con el conjunto de conceptos o imágenes recién señaladas.

Sirva como primer ejemplo la noción de "campo". Aunque los trabajos investigativos de Fuentes (1998), Vidales (2011), Jensen (2012a; 2012b), Moragas (2011) o Peza (2013) tiendan a referirla desde el orbe teórico de Pierre Bourdieu (1976), específicamente como "campo científico" o "espacio objetivo donde se encuentran comprometidas posiciones científicas" (p. 15), es decir, como un campo de fuerzas relativamente independiente y aislado donde se disputa el poder académico, esto no obsta un aspecto toral: esos mismos ejercicios reflexivos no abordan por exclusión los aspectos institucionales constitutivos del campo, sino antes aun los perfiles epistemológicos que los uncen; esto es, un conjunto específico de saberes y procedimientos que les ofrece un semblante científico. Siguiendo la línea semántica que ofrece la Real Academia Española, el origen etimológico descrito por Varrón (1990, libro v, 6, 3) y la propia construcción de Bourdieu, "campo" aparece como un espacio acotado que resguarda y preserva lo que ahí fructifica. Luego, desde los estudios de la comunicación, "campo" referiría a un constitutivo ontológico cerrado de conocimiento y prácticas académicas de la comunicación que choca con la evidencia de que no existe tal emplazamiento (Piñuel-Raigada, 2011), muestra la aspiración disciplinaria en el empleo del término y contraviene el sentido del llamado a la apertura de las ciencias.

Del mismo modo, las obras de Martín-Barbero $(2002,2010)$ han puesto de relieve los constructos teóricos de "mapa" y "cartografía" como un gesto literario que hace eco de Verne, Conrad, Saint-Exupéry, Stevenson o Kipling quienes, a su vez, elaboraron exuberantes mitologías que correspondieron literariamente a Magallanes, Cook o Amundsen. Es una bella idea incluso si se descarta el supuesto de que estos personajes implícitamente entrevieron en

\footnotetext{
${ }^{1}$ Sirvan los títulos referidos en la bibliografía como un ejemplo de esta afirmación.
} 
la aventura una forma moderna de conquistar el mundo y un derrotero espiritual que impondría el orden "natural" sobre los destinos de los hombres, particularmente si esos hombres son diferentes. Resulta, por ello, problemática la idea de armonizar la aventura intelectual que este autor nos conmina a recorrer con el espacio absolutamente otro -carente de sentido, de territorialidad, de coordenadas- que implica el hecho de tener un mapa, así sea bizantino, en la mano.

\section{EL CONOCIMIENTO CLAUSURADO: LA DISCIPLINA}

Edgar Morin (s. f.) recuerda que la palabra "disciplina" en uno de sus orígenes designaba al pequeño látigo de varias cuerdas con que en la antigüedad los clérigos se mortificaban. Queda en esta imagen la reminiscencia de las implicaciones reformatorias que atraviesan el aprendizaje y la adscripción a un campo disciplinado del saber. Así, aunque en las ciencias "la organización disciplinaria fue instituida en el siglo XIX" (Morin, s. f., p. 9), tendría que remontarse la búsqueda del sistema punitivo y metódico de estudio y aprendizaje, no sólo en las celdas monacales, sino en el amplio espectro de las tecnologías del cuidado de sí que la obra de Foucault detalla (2001a, 2001b). En este orden de ideas, la inter, multi, trans o posdisciplinariedad son convocadas -con diferentes alcances y enfoques-como una revuelta contra el disciplinaje del pensamiento, la visión unidimensional de los objetos y la inmutabilidad de las subjetividades.

Este conjunto de apuestas por la revolución del pensamiento no logran sin embargo desprenderse de dos aspectos muy importantes que prohijaron al sistema acotado de los saberes disciplinares. Uno es la trama epistemológica de orden cartesiano "Sujeto-Objeto" (Agamben, 2004) que Fried-Schnitman (1994) reconoce como meras construcciones sociales y que Colli ${ }^{2}$ ahonda hasta su disolución. Otro es la reticencia a abandonar, por un lado, el constructo moderno y humanista de Autor como lugar donde la cadena histórica y dialéctica de saberes se sintetiza positivamente (Sloterdijk, 2006a); y, en sentido

${ }^{2}$ En Filosofía de la expresión (2004), el filósofo italiano G. Colli propone un complejo entramado de tensiones donde el "mundo de las cosas no sería más que una concatenación, una estructura cognoscitiva" (p. 40), de modo que "el conocimiento existe, pero no hay un portador del conocimiento" (p. 43): las representaciones contienen al sujeto, pero no son creadas por él. 
inverso, apelar la Auctoritas como una estrategia simbólica para asegurarse un lugar en el campo académico (Bourdieu, 1976) que se evidencia, por ejemplo, en los sistemas de citación contemporáneos.

Aunque el rastreo de la razón positiva podría retrotraerse hasta las utopías de Moro, fue Descartes quien dio forma y fondo al proyecto ilustrado que forjó las disciplinas sociales del siglo XIX como sistemas cerrados de construcción y apropiación de objetos científicos. Esto se debe a que el cogito cartesiano se funda en una sustitución arbitraria de lo trascendental por lo lingüístico y, dice Agamben (2004), "el pensamiento moderno se ha construido sobre esa aceptación no declarada del sujeto del lenguaje como fundamento de la experiencia y del conocimiento" (p. 63). Este presupuesto asume que el sujeto antecede a la experiencia; claro está que este sujeto es trascendental y, por tanto, literalmente, sin existencia o lugar en el mundo de los accidentes.

La Ilustración francesa encontrará en la racionalidad cartesiana y la sintética resolución newtoniana al orden físico del universo, el modelo general de que la vida, la naturaleza y el mundo social son un rompecabezas, que "nos hallamos entre los fragmentos dislocados de este rompecabezas, y debe de haber un modo de poner estas piezas en su lugar" (Berlin, 2000, p. 45). Ese modo fue la razón positiva, su técnica fueron las disciplinas, y su metarrelato la Historia: principio toral de esta forma de construir y resolver las ideas como un relato unívoco del ser (Xirau, 1975) y como una lógica dialéctica que supedita trascendentalmente el destino de los individuos.

Fue este tono ilustrado de la razón el que alimentó a las ciencias sociales y puso el suelo fértil para el nacimiento y la institucionalización de los estudios de la comunicación. Como resultado de ello, tal como en las piezas de un rompecabezas, se establecieron fronteras, marcos o campos teóricos y metodológicos que integran mediante parcelas de conocimiento al orbe social que se asume como un conjunto lógico de piezas cuyas aristas corresponden entre sí. No obstante, por lógico que parezca, este tipo de racionalidad fue cuestionada por la propia historia que desdijo -en los campos de exterminio nazi, la posmodernidad, la globalización o los actuales peligros ecológicos- los proyectos utópicos que el pensamiento disciplinar simboliza. El cuestionamiento se establece al menos en tres frentes que obligan a imaginar una racionalidad allende las fronteras, marcos o mapas de autoidentificación disciplinaria: la necesaria indeterminación de la lectura de la historia, de los sujetos que experimentan su vida comunicativamente y del observador. 
Entre las distintas descripciones de la posmodernidad prevalece la idea de que los grandes horizontes de comprensión o metarrelatos se han roto (Fehér, 1998; Harvey, 2004; Jameson, 1996); es decir, que el continuum temporal o dialéctica de los hechos sociales del pasado se ha destruido y que se ha de reinterpretar su sentido, no en la perspectiva de una lectura unívoca que explique lógica y causalmente nuestro presente, sino tal como lo señala Peters (1999) en su interpretación de Benjamin: "the mystical sources wifty dreaming but have shrewd relevance to concrete concerns. The present becomes intelligible as it is alligned with a past moment with wich it has a secret affinity" (p. 3). La historicidad es una alternativa hermenéutica y constructivista del tiempo histórico que ahonda el subsuelo tectónico de los hechos sociales para encontrar relaciones teóricas que reconocen a los objetos de estudio en una tirantez temporal y ontológica necesariamente incompletas: $\mathrm{Si}$ la disciplina narra el relato omnisciente de fenómenos científicamente "puros", se trataría de meditar una reflexividad que relate la historia de un fenómeno que no acaba de terminar.

En segundo lugar, así como el cogito cartesiano expropia la experiencia de los sujetos (Agamben, 2004), la epistemología positiva que deriva en los marcos disciplinares tiende a desdibujar su capacidad actante. Aunque la estructura ontológica heideggeriana del Dasein o la parole del modelo lingüístico de Saussure ya poseen elementos que piensan a un sujeto capacitado para comunicar electivamente y darse un lugar proyectivo y preposicional en las tensiones estructurales, los estudios de la comunicación hubieron de esperar hasta que las anteojeras ideológicas entraran en crisis para que se naturalizara la evidencia de que el estudio de los hechos comunicativos involucra a un conjunto multidimensional de saberes que pone en entredicho a los acercamientos de corte disciplinar.

Por último, otro elemento que cuestiona al pensamiento disciplinar es el lugar que ocupa el observador en la construcción del objeto de estudio. Kant y Nietzsche criticaban desde el siglo xIx el sistema epistémico de "verdad" emanado del modelo objetivo cartesiano que hace corresponder al objeto con la idea. Citado por Glasersfeld, Foerster es lapidario al respecto: "La objetividad es la ilusión de que las observaciones pueden hacerse sin un observador" (Watzlawick y Krieg, 1989, p. 19).

Dentro del panorama de los estudios de la comunicación hay que recordar que los trabajos de Peters y Pasquali representan un distanciamiento respecto de los marcos o mapas disciplinares que cobijan la autoidentifiación 
del campo. La forma en que Peters (1999) dispone la historia de la comunicación en Speaking into the air -rastreando el sentido ínsito de lo comunicacional en motivos históricos como el eros platónico, la Biblia, el ángel medieval o las posguerras del siglo $\mathrm{xx}$ - no es tanto para fortalecer la disciplina dentro de una narativa causal cuanto para ensanchar los límites disciplinares y mostrar rasgos de una multidimensionalidad teórica desde donde se pueden estudiar los actos comunicativos. Más crítico de la disciplina es Comprender la comunicación, obra donde Pasquali (2008) denuncia y desmonta el carácter positivo, sistémico y mediático que gobierna sobre los estudios de la comunicación; ello en vías de proponer una Teoría General de las Comunicaciones, sostenida en el pensamiento relacional de cuño kantiano, en la ética y en el diálogo como la forma de comunicación por excelencia -en este caso contrario a Peters (1999), quien postula a la diseminación como la forma comunicativa más justa.

\section{LA COMUNICACIÓN COMO EL ESPACIO RELACIONAL DEL CONOCIMIENTO}

Se ha visto cómo a pesar de que existe la conciencia acerca de la necesidad de abrir los escenarios disciplinares de las ciencias sociales y los estudios de la comunicación, pareciera que a través recursos lingüísticos ("campo", "mapa") o persistencias epistemológicas ("sujeto-objeto", "autor", "auctoritas") el imaginario de lo clausurado aparece todavía en el ethos de la investigación académica. Una pieza clave para imaginar un escenario diferente de las prácticas investigativas y de la manera en que la comunicación es pensada es retomar la analogía espacial para figurar su reflexión en los espacios abiertos de una racionalidad no disciplinarizante. Esto supondría, primero, el ejercicio de una razón sin campo ni mapas y, segundo, el bosquejo del pensamiento comunicacional como el espacio relacional del conocimiento.

Ante el riesgo de que la reflexión se convierta en un reflejo, ya no por la falta de reflexividad en el ojo del observador, sino por la propia ficción del lenguaje que hace pasar como "otro" lo que es propio -tal como parecería suceder en los términos "campo" y "mapa"- en El pensamiento del afuera, Foucault (2000) advierte la necesidad de reconvertir el lenguaje reflexivo: "Hay que dirigirlo no ya hacia una confirmación interior -hacia una especie de certidumbre central de la que no pudiera ser desalojado más- sino más bien hacia un extremo en que necesite refutarse constantemente: que una vez que 
haya alcanzado el límite de sí mismo, no vea surgir ya la positividad de lo que contradice sino el vacío de lo que va a desaparecer”. (p. 24).

Esta reconversión imprime el sello de lo que finalmente este artículo quiere explorar: la disciplinarización ha sustraído, incluso al nivel discursivo, el propósito originario de la actividad intelectual: pensar cobra fuerza y sentido en la meditación de lo impensado (Heidegger, 2005). Los referentes acerca de la reflexión y el discurso sobre la experiencia del afuera no aluden sólo un gesto posmoderno o posestructuralista aislado para destruir la razón cartesiana, humanista o disciplinaria del siglo xx. Los trabajos de Lévinas (1997, 1999, 2000), Sloterdijk (2000, 2006a, 2006b), Agamben (1995, 2004), Colli (2004, 2009), Bataille (1981, 2005), García Ponce (2001) o, en el marco de los estudios de la comunicación, Pasquali (2008), actualizan a manera de advertencia la centralidad positivista -ahora mutada en la razón técnica o de fines (Heidegger, 2003, 2005)- como la pérdida del sentido primario del pensamiento crítico:

De una novela es posible, en el límite, aceptar que la historia que en ella debía contarse al cabo no se cuente; pero de una obra crítica se suelen esperar en cambio resultados o, por lo menos, tesis que demostrar $y$, como suele decirse, hipótesis de trabajo. Y sin embargo, cuando la palabra hace su aparición en el vocabulario de la filosofía occidental, crítica significa más bien indagación sobre los límites de la conciencia, es decir sobre aquello que precisamente no es posible ni asentar ni asir (Agamben, 1995, p. 9).

Los afanes epistemológicos ilustrados o deslices lingüísticos disciplinarios en las ciencias humanas y sociales acotan la potencia soberana, fantasmática ${ }^{3}$ crítica del pensamiento en aras de legitimar un conjunto de argumentos dentro de los espacios de las convenciones de las instituciones científicas, de obtener un lugar en el mapa del campo, de construirse -en el caso de los estudios de la comunicación- un sistema identitario basado en pensamientos repensados que le han conferido una imagen espectacular y banal alineada con la instrumentalidad de su conocimiento (Fuentes, 2010).

${ }^{3}$ El propio Agamben $(1995,2004)$ describe la importancia epistémica que tuvo -desde los griegos hasta el mundo medieval- la capacidad de elaborar phantamas o imágenes que aseguraban la posesión de la experiencia cognitiva. La modernidad expulsó a la imaginación como herramienta científica y expropió a la experiencia en forma de experimentación. De ahí que, por ejemplo, el científico moderno no experimente su objeto, pero sí pueda experimentar con él. 
Imaginar a la comunicación, ya no como un campo de saberes constitutivos, sino como la apertura de un espacio relacional del conocimiento, recoge parte de estas inquietudes y explora la potencia impensada de su papel central para articular comprensivamente las formas en que los procesos informativos integran, tensan o desaparecen las formas simbólicas que estructuran las relaciones humanas y el modo en que hermenéuticamente nos hacemos un lugar en el mundo.

Considerado desde la lógica saussureana, el campo o emplazamiento corresponde con el sistema gramatical de una langue que dota a los sujetos de un equipaje hermenéutico necesario para interpretar el conjunto de referencias -simbólicas, culturales, históricas, coyunturales- que los coloca interpretativamente frente al orbe de los fenómenos y sus pares comunicativos comprendidos desde el lugar que ocupan dentro del sistema; también invoca, como se observaba antes, una espacialidad acotada. El espacio, por otro, guarda un sentido más genérico y abierto; es una analogía de la parole o práctica electiva dentro del sistema estructurado de relaciones que, al contrario del emplazamiento, no está cerrado para que también aparezca la otredad: los otros sujetos practicando interpretativamente la ajena forma de comprender su propia espacialidad. En este orbe, la comunicación no aparece ante los ojos como un cuerpo estudiado por sus condiciones físicas o químicas; tampoco lo hace como el número, la ley o el dinero en la mirada simbólica. La comunicación se concibe aquí como aquello que no es pero que hace que las cosas aparezcan transmisiblemente ante y dentro de los demás. Por ello es el pasaje para que los emplazamientos tengan lugar en los sujetos, para que estos participen subjetivamente en aquellos y para que los sujetos mismos acontezcan interpretativa y proyectivamente en el espacio abierto de las relaciones con los otros.

La proyectividad propia de la comunicación humana y social refiere la naturaleza hermenéutica y movimiento preposicional de la existencia que estableció Heidegger (1971) en una analítica existenciaria del dasein. El plano donde acontecen el conjunto de proyecciones o direcciones existenciales, decimos, es el espacio comunicativo (Barrón, 2004). Del modo como fue descrito en las anteriores líneas, es ciertamente un emplazamiento porque es restrictivo, ofrece sentido y podría caracterizarse con la preposición desde, pues es origen donde ocurren proyectivamente los hechos comunicativos.

Es restrictivo porque limita a las proyecciones comunicativas dentro de una determinada frontera hermenéutica que los impulsa: incluso en el mun- 
do de las mediaciones virtuales un mensaje se encuentra constreñido a un conjunto particular de rasgos idiomáticos desde donde es emitido y comprendido. La segunda característica deriva justo de la anterior restricción, dado que al delimitarse lingüística, social, cultural o temporalmente la infinitud hermenéutica, los fenómenos son sensibles de cobrar sentido y los sujetos - en su capacidad de construir las interpretaciones de los fenómenos- un lugar identitario en el mapa de las estructuraciones.

No obstante, estas necesarias condiciones estructurantes propias del "campo" de la comunicación, el espacio de la práctica comunicativa es donde los sujetos se transforman en direcciones existenciales: dimensión abierta donde el acto de producir socialmente sentido es liberado. El espacio relacional en el que finalmente tiene lugar el encuentro de las distintas y multidimensionales direcciones de existencia. Si el "campo" es el "desde", el "espacio relacional" es el "hacia": el mundo de los otros que pone en juego el carácter ético y ontológico (Lévinas, 1997, 1999, 2000) de las manifestaciones humanas y sociales. La condición comunicativa del modo de acontecer lo humano es también una condición ontológica pues existir es colocarse interpretativamente en el abierto plexo del mundo (Heidegger, 1971); de ahí que la comunicación también ha de pensarse allende las fronteras restrictivas de lo dado, pues ella misma implica una indagación crítica sobre aquello que precisamente no es posible ni asentar ni asir; es decir, el rostro del otro al que la comunicación le confiere un asilo interpretativo.

Para concluir, y a manera de síntesis, en este artículo se han mostrado particularmente tres ideas en torno del estudio de la comunicación, en tanto disciplina:

1. Frente a los llamados a cuestionar y abrir los marcos teóricos y supuestos epistémicos heredados por la modernidad ilustrada, el empleo de la noción de "campo" entre los estudios de la comunicación es contradictoria debido a que el propio término refiere un constitutivo cerrado de conocimiento.

2. Hay horizontes de conocimiento que cuestionan y critican a los modelos epistémicos e históricos de la noción de "disciplina" y del pensamiento que la configura. En los estudios de la comunicación son ejemplares los casos de Peters (1999) y de Pasquali (2008).

3. Un modo de imaginar una racionalidad sin campo y no disciplinarizante dentro de los estudios de la comunicación, es pensar a la comunicación como la apertura de un espacio relacional del conocimiento. 
Bien se ha dicho en múltiples sitios que "comunicación" es un concepto polisémico, de difícil comprensión y confusa construcción teórica. Ello significa una cosa: está viva y todavía no se ha documentado apropiadamente su paso por la historia de las ideas. Que esta vitalidad caótica no sea un pretexto para abandonar los riesgos de su meditación y la responsabilidad histórica de asumirla como el instrumento inefable con el que el orbe social vive en los sujetos y, todavía más importante, con el que los individuos irrumpen e interrumpen el mundo.

\section{LISTA DE REFERENCIAS}

Agamben, G. (1995). Estancias, La palabra y el fantasma en la cultura occidental. Valencia: Pre-textos.

Agamben, G. (2004). Infancia e historia. Buenos Aires: Adriana Hidalgo.

Barrón, G. (2004). Hacia una teoría del espacio comunicativo: Una lectura ontológica de la acción comunicativa (Tesis inédita de maestría). Universidad de Guadalajara, México. Recuperado de https://ccdoc.iteso.mx/acervo/cat. aspx?cmn=browse\&id=4660

Bataille, G. (1981). El Aleluya y otros textos. Madrid: Alianza.

Bataille, G. (2005). Escritos sobre Hegel. Madrid: Arena.

Berlin, I. (1992). Árbol que crece torcido. México: Vuelta.

Berlin, I. (2000). Las raíces del romanticismo. Madrid: Taurus.

Bourdieu, P. (1976). Los usos sociales de la ciencia. Buenos Aires: Nueva Visión,

Bourdieu, P. (2012). La causa de la ciencia. Cómo la historia social de las ciencias sociales puede servir al progreso de estas ciencias. En Intelectuales, política y poder. Madrid: Clave Intelectual-EudeBA.

Colli, G. (2004). Filosofía de la expresión. Madrid: Siruela.

Colli, G. (2009). La naturaleza ama ocultarse. México: Sexto Piso.

Craig, R. T. (1999). Theorizing communication. Readings across traditions. Thousand Oaks: Sage.

Fehér, F. (1998). Políticas de la posmodernidad. Ensayos de crítica cultural. Barcelona: Península.

Foucault, M. (2000). El pensamiento del afuera. Valencia: Pre-Textos.

Foucault, M. (2001a). Dits et écrits I, 1954-1975. Gallimard: París.

Foucault, M. (2001b). Dits et écrits II, 1976-1988. Gallimard: París.

Fried-Schnitman, D. (comp.) (1994). Nuevos paradigmas, cultura y subjetividad. Buenos Aires: Paidós. 
Fuentes, R. (1998). La emergencia de un campo académico: continuidad utópica y estructuración científica de la investigación de la comunicación en México. Guadalajara: ITEso/ Universidad de Guadalajara.

Fuentes, R. (2002). Comunicación, cultura, sociedad: fundamentos conceptuales de la posdisciplinariedad. En Tram(p)as de la comunicación y la cultura. Mar del Plata: Universidad Nacional de La Plata.

Fuentes, R. (2003). La producción social de sentido sobre la producción social de sentido: hacia la construcción de un marco epistemológico para los estudios de la comunicación. En Epistemologia da Comunicação. São Paulo: Loyola (Comunicação Contemporânea, 1).

Fuentes, R. (2010). Investigación de la comunicación: referentes y condiciones internacionales de un diálogo transversal de saberes. Signo y Pensamiento, 29(57), 38-48. DOI: https://doi.org/10.11144/Javeriana.syp29-57.inco

Fuentes, R. (2011a). 50 años de investigaciones de la comunicación en México: un recuento descriptivo de la producción publicada. Intercom: Revista Brasileira de Ciências da Comunicação, 34(1), 213-231. DoI: https://doi.org/10.1590/ S1809-58442011000100011

Fuentes, R. (2011b). Condiciones institucionales para la práctica de la investigación académica de la comunicación: la persistencia de la triple marginalidad en México. En Qué pasa con el estudio de los medios. Diálogo con las ciencias sociales en Iberoamérica. Zamora, España: Comunicación Social.

García-Ponce, J. (2001). La errancia sin fin. Barcelona: Anagrama.

Harvey, D. (2004). La condición de la posmodernidad. Buenos Aires: Amorrortu.

Heidegger, M. (1971). El ser y el tiempo. México: FCE.

Heidegger, M. (2003). Camino de campo. Barcelona: Herder.

Heidegger, M. (2005). ¿Qué significa pensar? Madrid: Trotta.

Jameson, F. (1996). Teoría de la posmodernidad. Madrid: Trotta.

Jensen, K. B. (2012a). The cultural contexts of media and communication. En A handbook of media and communication research. Qualitative and quantitative methodologies. Londres/Nueva York: Routledge.

Jensen, K. B. (2012b). The humanistic sources of media and communication research. En A handbook of media and communication research. qualitative and quantitative methodologies. Londres/Nueva York: Routledge.

Jensen, K. B. (2012c). The social origins and uses of media and communication research. En A handbook of media and communication research. Qualitative and quantitative methodologies. Londres/Nueva York: Routledge. 
Krippendorff, K. (1994). The past of communication's hoped-for future. En M. R. Levy y M. Gurevitch (eds.), Defining media studies. Reflections on the future of the field. Oxford/Nueva York: Oxford University Press.

Lévinas, E. (1997). Totalidad e infinito. Salamanca: Sígueme.

Lévinas, E. (1999). De otro modo que ser o más allá de la esencia. Salamanca: Sígueme.

Lévinas, E. (2000). Sobre Maurice Blanchot. Madrid: Trotta.

Martín-Barbero, J. (2002). Oficio de cartógrafo. Travesías latinoamericanas de la comunicación en la cultura. Santiago de Chile: FCE.

Martín-Barbero, J. (2010). Preámbulo a un mapa de las mutaciones comunicativas y culturales. En De los medios a las mediaciones. Comunicación, cultura y hegemonía. Barcelona: Anthropos Editorial/uAm-Azcapotzalco.

Moragas, S. M. de (2011). Interpretar la comunicación. Estudios sobre medios en América y Europa. Barcelona: Gedisa.

Morin, E. (s. f.). Sobre la interdisciplinariedad. Icesi.

Ortiz, R. (1999). Ciencias sociales, globalización y paradigmas. En R. Reguillo y R. Fuentes (coords.), Pensar las ciencias sociales hoy. Reflexiones desde la cultura. Guadalajara: ITESO.

Pasquali, A. (2008). Comprender la comunicación. Barcelona: Gedisa.

Peters, J. D. (1999). Speaking into the air: A history of the idea of communication. Chicago y Londres: The University of Chicago Press.

Peza, C. de la (2013). Los estudios de comunicación: disciplina o indisciplina. Comunicación y Sociedad, 20, 11-32. Dor: https://doi.org/10.32870/cys.v0i20.215

Piñuel-Raigada, J. L. (2011). La docencia y la investigación universitarias en torno a la Comunicación como objeto de estudio en Europa y América Latina. La Laguna, Tenerife: Sociedad Latina de Comunicación Social (Cuadernos Artesanos de Latina, 15).

Pitkin, H. (1984). Wittgenstein: el lenguaje, la política y la justicia. Madrid: Centro de Estudios Constitucionales.

Reguillo, R. (2004). Los estudios culturales. El mapa incómodo de un relato inconcluso. PortalComunicación.com. Recuperado de https://incom.uab.cat/portalcom/ wp-content/uploads/2020/01/16_esp.pdf

Sloterdijk, P. (2000). El pensador en escena. Valencia: Pre-Textos.

Sloterdijk, P. (2006a). Normas para el parque humano. Madrid: Siruela.

Sloterdijk, P. (2006b). Venir al mundo venir al lenguaje. Valencia: Pre-Textos.

Varrón, M. T. (1990). De lingua latina. Barcelona: Anthropos.

Vidales, C. E. (2011). El relativismo teórico en comunicación. Entre la comunicación como principio explicativo y la comunicación como disciplina práctica. Comunicación y Sociedad, 16, 11-45. DoI: https://doi.org/10.32870/cys.v0i16.1102 
Wallerstein, I. (2004). The uncertainties of knowledge. Philadelfia: Temple University Press.

Watzlawick, P. y Krieg, P. (1989). El ojo del observador. Barcelona: Gedisa.

Xirau, R. (1975). El desarrollo y las crisis de la filosofía occidental. Madrid: Alianza.

Zallo, R. (2011). Retos actuales de la economía crítica de la comunicación y la cultura. En L. A. Albornoz (comp.), Poder, medios, cultura. Una mirada crítica desde la economía política de la comunicación. Buenos Aires: Paidós. 